\title{
33
}

\section{Improving cooperative behaviour in a multi-agent system}

\author{
O. Štěpánková, V. Mařík, J. Lažanský
}

Czech Technical University, Faculty of Electrical Engineering, Technická 2, CZ-166 27 Prague, Czech Republic

Phone: (+42 2) 293107, Fax: (+42 2) 290159

E-mail: \{step, marik, lazansky\}@lab.felk.cvut.cz

\begin{abstract}
The paper briefly describes DISCIM - an environment for experiments in multi-agent systems characterized by peer-to-peer communication among all agents and including a super-agent watching the communication traffic within the system. There are identified some features of the agents and their community which seem to be decisive from the point of the system's efficiency as well as the methods how to learn them from experimental data taken from the system's history.
\end{abstract}

\section{Keywords}

Multi-agent systems, machine learning, system reconfiguration, community behaviour

\section{DISTRIBUTED PROBLEM SOLVING}

The tasks of computer supported decision making are becoming more and more complex and cannot be solved by monolithic software systems. The extent and diversity of knowledge required to achieve acceptable results is growing rapidly.

All these facts cause that modular, distributed solutions as well as open system architectures are in the focus of software engineers' attention.

From the point of view of Artificial Intelligence there are two possible approaches to the task of distributed problem solving, namely

- distributed systems communicating via blackboard as a shared control and data structure,

- multi-agent systems consisting of autonomous entities communicating among themselves in a 'peer-to-peer" mode by message passing (with no centralised memory or control) allowing for intermediate result and task sharing.

The multi-agent architecture proved to be more open. It enables to make structural changes in a simpler way. That's why this architecture is currently preferred by many software system designers.

Current literature on computer science considers an agent as an object in the OO paradigm sense having a well defined interface for communication through messages with other agents. Each agent is able to respond to requests of other agents while exhibiting some goal-directed behaviour and requesting aid from other members of 
the agents' community. There are attempts to define an agent communication language (ACL) which should meet the following requirements:

- ACL must be general enough to enable communication among differently specialised agents.

- It must be open to enable enhancement of its expressing power.

- ACL should make it possible to transfer different types and formats of data.

- ACL should provide mechanisms to transfer data processing methods that will execute on various computing platforms hosting the agents.

All current ACL specifications are based on the idea of declarative statements exchanged between the agents. The commonly discussed standard for this communication level is based on the KQML (Knowledge Query and Manipulation Language) philosophy (Finin, 1995).

KQML is, in fact, an external language describing the expressions that compose the ACL. The internal language of describing the format of the expressions is the KIF (Knowledge Interchange Format); it is already standardised by ANSI and the ISO standardisation is expected soon. KIF is a prefix version of the first order predicate calculus with some features added for enhanced expressiveness. The entire inter-agent communication mechanism is based on a domain and task specific dictionary of terms that are well understood by the communicating agents.

The main problem of the current research in this area is that limited practical experience on practical implementations and overall agents' community behaviour is available. That is why our approach to treatment of multi-agent systems has been strongly experimentally oriented with the aim to study how a community of agents can behave and what are the issues for improving the overall behaviour.

\section{DISCIM}

\subsection{Basic Properties and Design Principles}

The initial goal of our activities has been to design and implement a programming environment for creating multi-agent decision support systems consisting of geographically distributed highly diverse units (agents). The resulting environment called is DISCIM and the following principles have been used to design it:

a) The agents are independent, autonomous entities communicating in the peer-topeer way among themselves. The asynchronous message passing/broadcasting in UNIX/INTERNET environment is used to perform this communication.

b) Each agent consists of a functional body (usually a stand-alone program with a well-defined functionality) and a wrapper (which is responsible for involvement of the agent into the community of agents). The wrapper contains a model of the agent's behaviour in the form of the list of classes in the Eiffel notation. The class descriptions, of course, contain messages which the agent can receive and send out. As a matter of fact, the wrapper translates the inter-agent communication into the instructions for the activity of the functional body and mediates the results of the body activity back to the agents' community.

c) The simplest typical model of agent's behaviour contained in the wrapper (Mařík, 1996) describes only the reactive part of behaviour. The deliberative behaviour 
which relies on explicit. internally held symbolic models (if such behaviour is required) is expected to be implemented as a natural part of the agent's body.

d) There is no central part of memory or control in the agents' community. Similarly to ARCHON (Jennings ,1993), the corresponding pieces of the control strategy are 'owned' by the individual agents, they are embodied into the individual behavioural models as rules contained in the classes (input/output messages description).

e) There is a library of standard classes/messages (written in the OO-oriented programming language Eiffel) available for the environment user (= application designer). This library, called DISCIM library, helps him/her to create his/her own agents by very fast agentification of arbitrary stand-alone programs.

f) User interface has been designed as a separate agent in the community. It is not only an agent responsible for communication with the user: It is the only agent which can initialise the activity of the agents' community. In some sense, it is a mobile agent as it can be run on each of the computers in the considered network. The mobility of this agent is an important feature providing high system flexibility from the end user's point of view. The activity of the whole system is expected to be initiated just by the user interface agent which is usually (but not necessarily) placed on the same machine as the most pro-active, so called 'root' agent in the community.

The basic types of agents currently developed and tested are:

- a rule-based diagnostic expert system agent,

- a database agent (as a body of which the POSTGRES OO-database is considered),

- a qualitative simulation agent using QUASIMODO system (Štěpánková, 1994),

- a user interface agent,

- the neural network agent running on a set of transputers is under development.

\subsection{Comparison with Other Multi-Agent Systems}

Comparing DISCIM to other multi-agent systems, several substantial differences should be mentioned. These differences result from the fact that DISCIM is a tool for intelligent, top-level decision making (neither operating system nor programming language for distributed computations).

DISCIM is intended to work with relatively few agents playing the role of welldeveloped stand-alone programs (the global system typically consists of several cooperating agents of diverse functionality only - the granularity of the system is very coarse). DISCIM is implemented on a higher programming level than different CORBA-based implementations of object manipulators (like ORBIX of IONA Ltd. or DSOM of IBM). DISCIM agents are more self-contained. This implies less frequent inter-agent communication.

The DISCIM communication language is general enough, written in Eiffel and supported by PVM (Parallel Virtual Machine) software. Its principal feature, which makes it different from KQML, is the fact that all the messages are broadcast. This enables to observe the situation in the agents' community from every node of the net.

The wrappers are also programmed in Eiffel which - in combination with PVM provides very efficient inter-agent communication facilities. We have carefully analysed the opportunity to reimplement DISCIM in JAVA, but our opinion is 
consistent with that presented by (Nygren, 1996): JAVA currently provides a weak inter-agent communication support.

Similarly as some other multi-agent systems (like fm-ASPECTS of Framework Technologies Ltd. or PELOROUS of Computervision Ltd.), DISCIM contains a set of pre-defined structures and classes usable in various application domains. DISCIM structures. however, form building blocks for composing task-oriented wrappers (together with some special wrappers themselves) thus enabling for design of standardised interfaces ('sockets') between the agent's body and its wrapper. These DISCIM standards have been designed for some specific decision-making bodies (see above) where several common requirements were detected. It is expected that the ongoing research will provide several standard socket types for a wider class of agent bodies.

This way DISCIM contains communication classes (and some task-oriented body applications) while the other mentioned multi-agent systems rely upon applicationoriented classes (like frames for declarative representation of manufacturing means and mechanical parts in fm-ASPECTS). The DISCIM units can be directly applied as modules for construction of particular problem-oriented applications preventing the necessity to solve the so called impedance problem for the agents.

The system GENIE (Toomey, 1996) uses similar philosophy, but the agents cannot communicate really peer-to-peer: The user agent's demands are transformed by the broker and matchmaker agents which later invoke the server agents. The reply of servers is again mediated by the broker and matchmaker agents. In this way, there might be constraints limiting the fully multi-agent behaviour.

In the other similar system PEACE+ (Latrous, 1996) each community also has to contain pre-specified types of agents - manager, launcher, broadcaster, reflector(s), enactor(s), etc. Each process agent must be connected with the broadcaster through a dedicated reflector and enactor agents. The lost of clear granularisation of the task into self-contained agents as well as the complicated system structure makes the system cumbersome and less transparent.

Although DISCIM has been designed mainly for integration of decision making systems at a high programming level, the experience gathered has wider consequences. It will provide motivation for introducing strategies for development of organisation within the system reflecting its specific needs, e.g. identification of

- clusters of nodes which should share their results,

- domain specific rules for task splitting and sharing.

Our agents are "workaholics"; the problems with selfish agents are not considered. Our utility function for task sharing is based primarily on time and space requirements.

\section{TOWARDS COOPERATIVE COMMUNITY OF AGENTS (Inspired By Social Reality)}

The agents in DISCIM represent well-developed stand-alone programs converted to agents by means of an agentification technology under development.

Many AI principles have been explored inside each of the agents. On the other hand, there are promising opportunities for applying AI principles in organising the inter-agent behaviour within the community. The following problems of cooperation will be discussed and the corresponding $A I$ solutions will be described in more detail: 
- Reconfiguration problem (Perestroyka). Very often, one or more of agents can loose the connection with the community or can be temporarily busy/overloaded. Such a situation has to be recognised first. System reconfiguration is required as a consequence. This results in creating new agents and relocation of the load inside the agents' community. In DISCIM, both problems are solved by means of a special agent (referred to as a super-agent) which is responsible for observing the message traffic and for taking specific actions (like creating and starting new processes) to ensure correct functionality of a community subset. There are usually more super-agents present in the community covering by their activity different possibly overlapping subsets of agents. The super-agents have the same nature and structure as the regular agents but their decision making is aimed at fault detection and community reconfiguration in critical situations. Machine learning methods are applied for this purpose.

- Problem solving based on bids and contracts (Market-Oriented Economy). The DISCIM communication language is rich enough to involve the bids and other negotiation messages. The models of behaviour owned by each of the agents can be easily extended to contain the negotiation algorithms based on the $\mathrm{AI}$ negotiation method presented in (Findler, 1995) aimed at the task sharing. The task division could be based on results obtained through a 'rough' (qualitative) model of a suitable problem solving process for the case at hand. Such a model can be developed by a qualitative simulation agent using model revision (Mařik, 1996) approach.

- The role of deep models. A meta-agent equipped with a tool for the qualitative or case-based reasoning can support even other tasks. It can be useful namely for the purpose of

- optimising the multi-agent system architecture to speed-up the decision making process (introduction of coalitions),

- simplification of agents' requests (message filtration - censorship),

- providing 'rough' global explanations (propaganda) of the physical background of the decision making to the user.

\subsection{Super-Agents}

The original idea behind DISCIM is to organise the agents as a community or society of mutually communicating and cooperating agents. No special internal community structure was considered. The idea of introducing the super-agents is not - in principle - in contradiction to the initial vision: As a matter of fact, the agents as well as superagents can freely communicate among themselves using the same language, they have the same internal structure (e.g., qualitative simulation nature or an expert system background etc.). The only difference is caused by the fact that the super-agents don't process external data. but the information on the activities and behaviour of the others. This is the case of a 'watching agent' (Hall, 96). The super-agents can be considered as regular agents processing the information on a higher gnoseological level.

The introduction of super-agents - sometimes called meta-agents or FUNs (Farhoodi, 1996) - reflects natural tendency to partially organise the society to improve its overall performance. This approach is aimed at creating of better organised subsets of units with better-informed higher-level agents which are able to evaluate the activities of the others, to re-distribute the tasks and optimise the 
activities. The trend of creating function-oriented institutions (like governments, companies etc.) guiding the activities of the society members with the goal to achieve better performance is clearly visible in each human society, too.

The machine learning principles are embodied as basic algorithms in some of the agents already developed within the DISCIM project. Why not to explore the same algorithms (or even the same agents) on the higher, 'more strategic' level to make the overall efficiency much better? Why not to use the same or similar programs which are able to learn rules from sets of external data to learn rules from the data describing the community behaviour?

\subsection{Machine Learning on the Super-Agent Level}

In (Elias, 1996), the authors analyse and summarise their experience in building distributed real-life applications in order to identify the main design principles that the designers are trying to follow when creating a distributed system:

- Allocate tasks to agents so as to minimise inter-agent communication and to maximise agent cohesion.

- Allocate independent tasks and tasks that compete for agent resources to different agents, exploiting concurrence and relaxing task dependencies.

- Allocate tasks to agents so as to allow human operators to identify decision centres and decision levels.

These principles aim to ensure optimal functionality of the resulting system. Sophisticated design techniques can help to reach this goal in a net of agents with fixed set of participants as well as fixed geometry characterised by the matrix of distances between any two agents. The crucial decisions which agent to include and how to place the agents in space are made in advance - the designer builds in all what is known about qualities of the individual agents and their mutual relations. In some cases, necessary information may be lacking or too complex to be of any use. Moreover, we have to consider a more realistic setting with a flexible environment where the available resources change in time (due to the load of the net, local limitations or extensions, etc.). Under such dynamically changing conditions the network should be able to adjust itself automatically to the new situation.

Machine learning has been studying the problems how to utilise experience to reach more efficient behaviour on tasks similar to those already solved. A number of algorithms has been developed for various environments and learning conditions. Can some of those be applied to ensure flexible adaptation of a distributed net of agents? Let us distinguish three types of observations which can be extracted from the past history of the agents community. These types correspond to the following central targets of observation: the single agent behaviour, a specific task to be fulfilled, or communication within the agents' community.

Each of these types will prove to be relevant for achieving a certain feature of adaptive behaviour of the entire system. It is certainly no surprise that characteristic properties of social systems will re-appear in this context. Let us try to identify those relevant features together with the methods and data that should be used to extract them.

i) Case of a single agent: Whenever a social system has to adjust to a new situation the cooperating partners are evaluated. Their reliability is considered to be one of the most important features then. The same is true for the set of artificial agents. In our context agent's reliability can be inferred from 
- the due dates the agent promises and later meets (or does not),

- answers the agent produces as a reaction to the same question.

Moreover, active presence of an agent in the community is a necessary condition fro any cooperation with it. We have to be able to identify as quickly as possible when there is something wrong with the agent, e.g. because there is an obstacle preventing its communication with the rest of the net. This can be inferred from the record informing about the recent agent's work load as well as about its average. The difference between the recent value and that one obtained from the long term data may point to an abnormality in the node's function.

ii) Case of a specific task: When we are concerned with a specific task we want to know the right answer as well as the agent who is the best agent to provide it. There is no way how to compare two answers unless there is an evaluation function for that purpose - it has to be a part of the problem definition. But to find the best agent for a given task we can use various criteria for evaluation of quality of a solution, namely the precision of the offered answer, its cost (expressed in terms of memory and communication resources used) and availability (how quickly it is produced). Often a task is not solved by the agent alone - further cooperating agents are invited to get involved when searching for answer to the initial question. This information is somewhat connected to the notion of its cost but it is worth to be stated explicitly, because availability of supporting nodes can be decisive when comparing bids of various agents. We are collecting data from the communication history as a data set structured as follows:

$$
\begin{aligned}
& \text { <specific_task, answer, agent_providing_the_answer, } \\
& \text { prize_of_the_answer, set_of_all_the_supporting_agents>. }
\end{aligned}
$$

Such a data set can be used to answer some presented tasks directly. If we have a similarity function for the tasks the collected data can serve to make a better (informed) choice during the biding period. On the other hand if no similarity function is available the collected data can help to define it using ML techniques - including ILP approach (Lavrač, 1994) allowing to take into account rich background knowledge - by characterising those tasks for which the same agent is the best one.

iii) Case of the inter-agent communication: The main danger for an agent community is its unintended looping during the search for an answer to a certain task. This can be identified from a sequence of all those tasks which have not been completed yet.

It is important to search for clusters of cooperating agents. A set corresponding to one of these clusters is a good candidate for restructuring, thus building 'close connection' in the near future of the net.

On the other hand co-appearing tasks are of importance as well. Is there a meaningful connection among the identified tasks? Could the answer to one of them be deduced from the other ones? Does it happen often that a more specific question is followed by a more general one? In such a case the system could spare some time if there is posed the general question only. Thus a certain change should be suggested leading to a new structure of the communication language in the net in order to reach more efficient conduct.

Problems of restructuring the description language of the training data have been tackled, e.g., by constructive induction (Muggleton, 1990) within ILP stream. Another consequence of existence of some co-appearing tasks should be considered in the case they represent queries to two different databases - then it might prove advisable to combine both in a new structure. 


\section{CONCLUSIONS}

We have mentioned several features which can be extracted from the net-history data collected by the super-agent. These features represent explicit application dependent knowledge about the dynamic behaviour of the considered multi-agent system. Similar approach has been suggested by (Hall, 1996) whose 'watching agent' searches for sources of inefficiency. The resulting knowledge provides a necessary ground for adaptive behaviour of the system. Moreover, a multi-agent system with a 'thoughtful' super-agent can be quickly build as a prototype of the target system - this prototype is understood to be an experimental environment to build the relevant task-dependent knowledge in the form immediately transferable into the final design of the system and its topology.

\section{REFERENCES}

Elias P., Chouraqui E. (1996) A Multi-Agent System for the Control of Pressurized Water Reactors in an Accidental Situation. In: PAAM (1996), 123-142

Farhoodi F., Graham 1. (1996) A Practical Approach to Designing and Building Intelligen Software Agents. In: PAAM (1996), 181-204

Findler, N. V., Elder, G. D. (1995) Multiagent Coordination and Cooperation in a Distributed Dynamic Environment with Limited Resources. Artificial Intelligence in Engineering, 9. 229-238

Finin T., et al. (1995) Specification of the KQML Agent Communication Language. Available from http://www.cs.umbc.edu/kqml/kqmlspec.ps

Hall T., Shahmeri N. (1996) An Intelligent Multi-Agent Architecture for Support of Precess Reuse in a Workflow Management System. In: PAAM, (1996), 331-344

Jennings N. R., Witting T. (1993) ARCHON: Theory and Practice. In: Distributed AI: Theory and Paxis (Avouris M., Gasser L., Eds.), Kluver Academic Publ. Dordrecht, 179-195

Latrous S., Oquendo F. (1996) A Reflective Multi-agent System for Software Process Enaction and Evolution. In: PAAM (1996), 853-870

Lavrač N., Džeroski S. (1994) Inductive Logic Programming. Ellis Horwood.

Mařík, V., Štěpánková, O.. Flek, O. (1996) Multi-agent System with Qualitative Simulation Unit. In: Proc. of the XIII European Meeting on Cybernetics and Systems Research, Vienna, 1183-1188

Muggleton S. (1990) Inductive Acquisition of Expert Knowledge. Turing Institute Press.

Nygren K., Jonsson I-M., Carlvik O. (1996) An Agent System for Media on Demand System. In: PAAM (1996), 437-454

PAAM (1996) Proceedings of the First Int. Conference on the Practical Application of Intelligent Agents and Multi-Agent Technology. The Practical Application Company Ltd., London

Štěpánková O. , Marvan I. (1994) Time Independent Global Constraints and QSIM. Proc. of ESM Conference, Barcelona, 470-474.

Toomey, Ch.. Mark, W. (1995) Satellite Image Dissemination via Software agents. IEEE Expert, vol. 10. No. 5, pp. 44-50 